\title{
Bestimmung des Moleculargewichtes der Cholalsäure, des Cholesterins und des Hydrobilirubins nach der Raoult'schen Methode
}

\author{
von \\ Dr. John J. Abel. \\ (Mit 1 Textfigur.) \\ Aus dem Laboratorium des Prof. Neneki in Bern.
}

(Vorgelegt in der Sitzung am 6. März 1890.)

Die in Nachfolgendem mitzutheilenden Bestimmungen habe ich ausgeführt, um zu ermitteln, inwiefern die Raoult'sche Methode bei hochmolecularen Verbindungen anwendbar ist, respective wie weit die Concentration der Lösungen gesteigert werden muss, um richtige Zahlen zn erhalten. Betrachten wir nämlich die Raoult'sche Formel,

$$
M=\frac{T \cdot P \cdot 100}{D . L},
$$

worin $M$ das Moleculargewicht, $T$ die moleculare Depression, $P$ das Gewicht der Substanz, $L$ das Gewicht des Lösungsmittels und $D$ die beobachtete Depression bedeutet, so wird bei wachsendem Werthe von $M$, die Werthe $T, P$ und $L$ als unverändert vorausgesetzt, die beobachtete Depression immer kleiner. Um nun die Erniedrigung des Erstarrungspunktes der Lösung (den Werth $D$ ) auf etwa $0.5^{\circ} \mathrm{C}$, zu treiben, wie dies Raoult fiur wässerige Lösungen verlangt, muss bei hochmolecularen Körpern der Werth $\boldsymbol{T}$ grösser oder $\boldsymbol{L}$. kleiner, d. h. die Concentration der Lösung erhöht sein. Vor Kurzem haben Nencki und Rotschy ${ }^{1}$ die interessante Streitfrage, ob die Zusammensetzung des Bilirubins

1 Sitzungsber. d. kais. Akad. d. Wiss., Bd.XCVIII, Abth. II. b, Juni 1889. 
der einfachen Formel $=\mathrm{C}_{16} \mathrm{H}_{18} \mathrm{~N}_{2} \mathrm{O}_{3}$ oder der verdoppelten $=\mathrm{C}_{32} \mathrm{H}_{36} \mathrm{~N}_{4} \mathrm{O}_{6}$ entspricht, mittelst der Raoult'sehen Methode zu entscheiden gesucht. Ihr Vorhaben kann kaum als gelungen angesehen werden, indem das Bilirubin in sämmtlichen hier in Betracht kommenden Lösungsmitteln zu wenig löslich ist, respective dadurch, wie z. B. durch Eisessig, verändert wird. Als das beste Lösungsmittel des Bilirubins erwies sich das Phenol. Eine gesättigte Lösung des Bilirubins in Phenol entbält etwa $0.4 \%$ des Farbstoffes und erst bei einem Gehalte von $0 \cdot 3-0 \cdot 4 \%$, entsprechend einem Molekül Bilirubin in 1000 Molekülen Phenol gelöst, ergaben die Bestimmungen der Formel $\mathrm{C}_{16} \mathrm{H}_{18} \mathrm{~N}_{2} \mathrm{O}_{3}$ entsprechende Werthe. ${ }^{1}$ Welche Depressionen, respective das daraus erhaltene Moleculargewicht erhalten worden wäre, wenn nicht 1 Moleküil, aber 2, 3 bis 10, z. B. in Phenol gelöst wären, das zeigen die Versuche von Nencki und Rotschy eben wegen der Unlöslichkeit dieses Farbstoffes nicht.

Es war wünschenswerth, festzustellen, ob Phenol sich ähnlich wie Eisessig verhält, von welchem $A$ uwers ${ }^{2}$ auf Grund seiner Beobachtungen angibt, dass die Depressionen nicht erst wenn dieselben einen halben Grad erreicht haben, anfangen normal zu werden, sondern dass Lösungen in Eisessig bereits von den kleinsten Erniedrigungen des Erstarrungspunktes dem Raoultschen Gesetze folgen. Ob diese Beobachtung Auwers' für alle Substanzen giltig ist, ist noch nicht erwiesen. Es war daher geboten, Substanzen von hohem Moleculargewicht anzuwenden, die einerseits hinreichend beständig, anderseits in den hier in Betracht kommenden Lösungsmitteln leicht löslich sind. Zwei solche Substanzen des Thierkörpers sind das Cholesterin und die Cholalsäure.

Die letztgenannte Verbindung, die Cholalsäure, habe ich mir selbst aus Rindergalle, mit einer kleinen Abänderung nach der Vorschrift von Mylius, ${ }^{3}$ dargestellt. Mylius kocht die Galle mit einem Fünftel ihres Gewichtes von $30 \% \mathrm{NaOH} 24$ Stunden unter Erneuerung des verdampfenden Wassers. Ich habe es vor-

1 Nencki und Rotschy, I. c. S. 548 .

2 Berichte der Deutschen chemischen Gesellschaft, Bd. 21, I, S. 708.

3 Zeitschrift für physiologische Chemie, Bd. 12, S. 262-266. 
gezogen, die mit der von Mylius angegebenen Natronlauge versetzte Galle in einem Autoclaven $3-3^{1 / 2}$ Stunden bei $130^{\circ}$ zu erhitzen. Durch wiederholtes Umkrystallisiren des gleich Anfangs krystallinischen Rohproductes erhielt ich das Alkoholat der Cholalsäure in Form von weissen, gut ansgebildeten Tetraëdern. Durch mehrmaliges Auflösen derselben in einer einprocentigen Natronlauge ${ }^{1}$ und Wiederausfällen mit verdünnter Salzsäure erhielt ich die freie Säure in reinem Zustande als amorphes Product. Wird solches Präparat Anfangs an der Luft, sodann über $\mathrm{H}_{2} \mathrm{SO}_{4}$ und hierauf zuerst 24 Stunden zwischen $90-100^{\circ}$ und dann 5 bis 6 Tage bei $120-130^{\circ}$ getrocknet, so erreicht es doch nicht ein constantes Gewicht. Die Schmelzpunktbestimmung der auf diese Weise getrockneten Säure zeigte, dass sie erst bei $174^{\circ}$ schmilzt. Dies stimmt mit der Angabe von Latschin off überein. Trocknet man wenjger lang, z. B. nur 2 Tage, so finde ich, dass die Säure bei $137^{\circ}$ anfängt $\mathrm{zu}$ sintern und bei $150-152^{\circ}$ vollständig geschmolzen ist. Trotzdem ergab die Elementaranalyse der durch Fällung mit Salzsäure dargestellten Cholalsäure nit der Formel $\mathrm{C}_{24} \mathrm{H}_{40} \mathrm{O}_{5}$ gut stimmende Zahlen. $0 \cdot 2425 \mathrm{~g}$ Cholalsäure gaben $0.6273 \mathrm{~g} \mathrm{CO}_{2}$ und $0.2146 \mathrm{~g} \mathrm{H}_{2} \mathrm{O}$, oder $70.54 \% \mathrm{C}$ und $9 \cdot 83 \% \mathrm{H}$. Die Formel $\mathrm{C}_{24} \mathrm{H}_{40} \mathrm{O}_{5}$ verlangt $70 \cdot 59 \% \mathrm{C}$ und $9 \cdot 80 \% \mathrm{H}$.

Das Alkoholat schmilzt bei $195^{\circ}$ und verliert $10 \cdot 3-10 \cdot 5 \%$ Alkohol beim Trocknen im Luftbade bei $110^{\circ}$ bis zur Gewichtsconstanz. Zum Beispiel, $2 \cdot 4941 \mathrm{~g}$ Alkoholat verloren $0 \cdot 2571 \mathrm{~g}$ an Gewicht, also $10.3 \%$, und $0.7512 \mathrm{~g}$ ver]oren $0.0788 \mathrm{~g}$, also ein Verlust von $10 \cdot 48 \%$. Die Formel $\mathrm{C}_{24} \mathrm{H}_{40} \mathrm{O}_{5}, \mathrm{C}_{2} \mathrm{H}_{5} \mathrm{OH}$ verlangt $10 \cdot 36 \%$ Alkohol.

Die freie reine Cholalsäure, sowie das Alkoholat sind in Eisessig und Phenol leicht löslich, weshalb ich auch meine Moleculargewichtsbestimmungen in diesen beiden Lösungsmitteln ausführte.

Den von mir benutzten einfachen Apparat verauschaulicht die beifülgende Zeichnung. Das Thermometer war von Go etze in Leipzig aus Jenaer Glas angefertigt, hat eine Scala von fünf Graden (Ziffern willkürlich), zu $1 / 100^{\circ}$ getheilt und ist am oberen Ende mit einem Quecksilberreservoir versehen. Das Thermometer

1 Schotten, Zeitschrift fïr physiologische Chemie, Bd. 10, s. 182. 
steckt durch einen luftdicht schliessenden, elastischen Gummikork in dem im Inneren die Lösung enthaltenden Gefässe $G$. An dem Korkring $\boldsymbol{R}$ wird der Mantel $\boldsymbol{M}$ befestigt. Statt eines Glas-

Fig. 1.

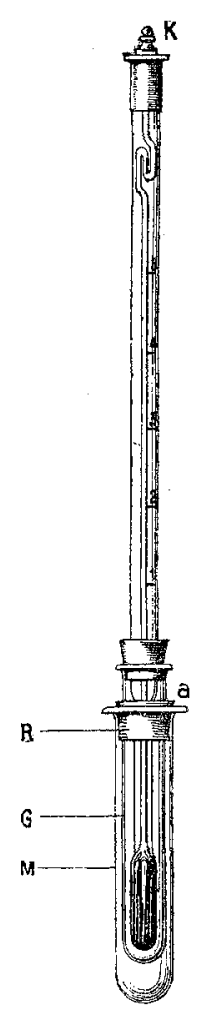

oder Platinriihrers wird die Lösung durch rotirende Drehbewegungen am Knopfe des Thermometers, $\boldsymbol{K}$, in Bewegung gesetzt. Durch den elastischen Kork wird die Bewegung am Knopfe der Quecksilberkugel mitgetheilt und dadurch noeh der Vortheil erreicht, dass bei bygroskopischen Lösungsmitteln, wie z. B. Eisessig, der Verschluss ein luftdichter ist. Diese Vorrichtung hat gegenüber dem kleinen Eykmann'schen ${ }^{1}$ Apparate den Vortheil, dass ein Thermometer mit feiner Eintheilung benutzt wird, und dass Lösungen von $10-50 g$, je nach der Grösse des inneren Gefässes $G$, durch die gleichmässige rotirende Bewegung (bei vollständig luftdichtem Verschlusse) sebr innig gemischt werden. In meinen Versuchen hatte das innere Gefäss ein Lumen von $2-2 \cdot 5 \mathrm{~cm}$, eine Länge von $13-15 \mathrm{~cm}$ und eine Wanddicke von $0.8-1 \mathrm{~mm}$.

Latschin off ${ }^{2}$ fand, dass die Cholalsäure in Phenol gelöst, sich damit zu cholalsaurem Phenolat verbindet, welches durch Zusatz von Benzol in schönen Krystallen gefällt wird. Die Krystalle, mit Benzol ausgewaschen, verändern sich nicht an der Luft. Latschinoff fand darin $71.55 \% \mathrm{C}$ und $9 \cdot 43 \%$ H. Beim Erwärmen auf $120^{\circ}$ verlieren die Krystalle Phenol. In drei Bestimmungen betrug der Verlust $16 \cdot 44 \%, 16 \cdot 64 \%$ und $16 \cdot 60 \%$. Die S tre cker'sche Formel des cholalsauren Phenolats $=\mathrm{C}_{24} \mathrm{H}_{40} \mathrm{O}_{5}, \mathrm{C}_{6} \mathrm{H}_{6} \mathrm{O}$ verlangt einen Verlust von $18 \cdot 72 \%$ Phenol, die Latschin off'sche Formel $=\mathrm{C}_{25} \mathrm{H}_{42} \mathrm{O}_{5}, \mathrm{C}_{6} \mathrm{H}_{6} \mathrm{O}$ einen Verlust von $18 \cdot 21 \%$. Sowohl die Zablen der von Latschin off mitgetheilten Elementaranalyse, sowie auch der Phenolverlust stimmen weder mit der Strecker'. schen, noch mit der Formel von Latschinoff gut überein. Die

1 Zeitschrift für physikalische Chemie, II, 12, 1888.

2 Berichte der Deutschen chem. Gesellschaft, Bd. 20, IV, 1887, S. 3278. 
Differenz in den theoretisch für die eine wie für die andere berechneten Werthe, von $\mathrm{C}, \mathrm{H}$ und Phenol ist jedoch nur gering. Ohne auf die Streitfrage einzugehen, ob die Strecker'sche Schreibweise oder die von Latschinoff vorgeschlagene die richtige sei, ist es doch am Platze, die Möglichkeit hervorzuheben, dass nicht Alles von der Cholalsäure in Phenolat verwandelt wurde. Latschinoff fand beim Verdunsten des Phenols einen durchschnittlichen Gewichtsrerlust von $16 \cdot 56 \%$. Nimmt man nun an, dass ein Molekül Phenol von einem Molekül der Säure gebunden wird, und dass das von Latschinoff gefundene Deficit an Phenol sieh nach der geäusserten Vermuthung erklären lässt, so enthielten 100 Gewichtstheile eines vermeintlichen Phenolats in Wirklichkeit nur $88.46 \%$ des Phenolats und $11.54 \%$ der nicht an Phenol gebundenen Säure (nach der Strecker'schen Formel gerechnet), nach der Schreibweise von Latschin off $90.93 \%$ des Phenolats und $9 \cdot 07 \%$ der freien Säure. Unter dieser Annahme würden die von Latschin off gefundenen Zahlen für $\mathrm{C}$ und $\mathrm{H}$ auch mit den für die Stre cker'sche Formel berechneten übereinstimmen. Dass die Übereinstimmung der auf diese Weise berechneten Werthe von $C$ und $H$ weniger gut ist für die von Latschin off erhaltenen Zahlen nach dem Verjagen des Phenols, dürfte nicht befremden, da man die zurückbleibende Säure erst wieder aus Alkohol umkrystallisiren sollte, bevor sie zur Analyse verwendet wird.

$\mathrm{Zu}$ den Moleculargewichtsbestimmungen benutzte ich die durch Trocknen des Alkoholats bis zur Gewichtsconstanz gewonnene freie Säure. Die freie Säure, welche durch Fällung des Natronsalzes mit $\mathrm{HCl}$ gewonnen wird, hält hartnäckig, wie schon eingangs erwähnt, $\mathrm{H}_{2} \mathrm{O}$ zurück, hat nicht einen constanten Schmelzpunkt und bräunt sich ein wenig beim langen Trocknen bei $120-130^{\circ}$. Aus diesen Gründen habe ich es vorgezogen, mit der aus dem Alkoholat durch Trocknen gewonnenen Säure zu arbeiten, trotzdem dass die mitgetheilte Elementaranalyse der durch Fällung gewonnenen Säure sehr gut mit der gebräuchlichen Formel tibereinstimmt.

Folgende Tabelle veranschaulicht die mit verschiedenen Concentrationen der Lösung gewonnenen Zahlen, wo Phenol als Lösungsmittel angewendet wurde. Das Phenol schmolz bei 
$41 \cdot 8^{\circ}$ und wurde von der Badisehen Anilin- und Sodafabrik als reines synthetisches Phenol bezogen. Da sich das Phenol mit der Cholalsäure verbindet, muss die eingangs gegebene Formel $M=\frac{T \cdot P .100}{D . L}$ in $M=\frac{T \cdot P+l .100}{D . L-l}$ verwandelt werden, worin $\boldsymbol{l}$ das Gewicht des von $\boldsymbol{P}$ gebundenen Phenols bezeichnet.

Für cholalsaures Phenolat, $\mathrm{C}_{24} \mathrm{H}_{40} \mathrm{O}_{5}, \mathrm{C}_{6} \mathrm{H}_{6} \mathrm{O}$, berechnet sich $M$ auf 502. Werden nur $88 \cdot 46 \%$ der Säure, als in Phenolat verwandelt, angenommen und der Rest von $11.54 \%$ der Cholalsäure als einfach gelöst, so würde der durchschnittliche Werth von $M$ gleich $491 \cdot 15$ sein.

Tabelle I.

\begin{tabular}{|c|c|c|c|c|c|}
\hline$D$ & $P$ & $L$ & $M$, & $\begin{array}{c}\text { Anzahl der } \\
\text { Procent- } \\
\text { gehalt der } \\
\text { Lösung }\end{array}$ & $\begin{array}{c}\text { Moleküle Cholal- } \\
\text { säure auf } \\
1000 \text { Moleküle } \\
\text { Phenol }\end{array}$ \\
\hline \hline & & gefunden & & \\
$0 \cdot 166$ & $0 \cdot 1300$ & $18 \cdot 46$ & 396 & $0 \cdot 7$ & $1 \cdot 6$ \\
$0 \cdot 278$ & $0 \cdot 2165$ & $16 \cdot 24$ & 449 & $1 \cdot 2$ & $2 \cdot 3$ \\
$0 \cdot 296$ & $0 \cdot 2085$ & $13 \cdot 15$ & 495 & $1 \cdot 4$ & $3 \cdot 81$ \\
$0 \cdot 336$ & $0 \cdot 2405$ & $13 \cdot 15$ & 509 & $1 \cdot 7$ & $4 \cdot 2$ \\
$0 \cdot 434$ & $0 \cdot 3778$ & $16 \cdot 90$ & 483 & $2 \cdot 18$ & $5 \cdot 2$ \\
$0 \cdot 84$ & $0 \cdot 715$ & $16 \cdot 10$ & 494 & $4 \cdot 19$ & $\mathbf{1 0} \cdot 2$ \\
1.410 & $1 \cdot 251$ & $17 \cdot 55$ & 472 & $6 \cdot 55$ & $\mathbf{1 6}$ \\
1.454 & $1 \cdot 344$ & $17 \cdot 83$ & 493 & 7 & $\mathbf{1 7} \cdot 3$ \\
& & & & & \\
\hline
\end{tabular}

Tabelle II.

Cholalsäure in Eisessig, $\mathrm{C}_{24} \mathrm{H}_{40} \mathrm{O}_{5}, M=408$.

\begin{tabular}{|c|c|c|c|c|c|}
\hline$D$ & $P$ & $L$ & $M$ & $\begin{array}{c}\text { Procent- } \\
\text { gehalt der } \\
\text { Lösung }\end{array}$ & $\begin{array}{c}\text { Anzahl der } \\
\text { Moleküle Cholal- } \\
\text { säure auf } \\
\text { 1000 Moleküle } \\
\text { Phenol }\end{array}$ \\
\hline $\begin{array}{r}0.55 \\
0.56 \\
0.27\end{array}$ & $\begin{array}{l}0.8265 \\
0.8832 \\
0.4472\end{array}$ & $\begin{array}{l}14 \cdot 88 \\
16 \cdot 5 \\
16 \cdot 5\end{array}$ & $\begin{array}{l}396 \\
372 \\
391\end{array}$ & $\begin{array}{l}5 \cdot 26 \\
5 \\
2 \cdot 63\end{array}$ & $\left.\begin{array}{l}8 \\
7 \cdot 7 \\
4\end{array}\right\}$ \\
\hline
\end{tabular}

1 Die eingeklammerten Werthe beziehen sich auf den gleichen Versuch, in welchem durch weiteren Znsatz von Substanz zu gleicher Lösung eine zweite Bestimmung ausgeführt wurde. 
Das von mir verwendete Cholesterin wurde früher im hiesigen Laboratorium ans menschlichen Gallensteinen erhalten und die Reinheit des Präparates durch die Elementaranalyse festgestellt. Ich habe das Präparat vor dem Gebrauche noch einmal aus Alkohol umkrystallisirt und bei $100^{\circ}$ getrocknet. Für Cholesterin eignet sich Eisessig als Lösungsmittel nicht, da schon eine geringe Menge Cholesterin, in Eisessig in der Hitze gelöst, noch bevor die Lösung erkaltet ist, zu einem Krystallbrei des essig. sauren Cholesterins, $\mathrm{C}_{26} \mathrm{H}_{44} \mathrm{O}, \mathrm{C}_{2} \mathrm{H}_{4} \mathrm{O}_{2}$, erstarrt. Ich habe die Bestimmungen daher nur in Phenol ausgeführt, worin das Cholesterin leicht löslich ist.

Tabelle III.

Cholesterin in Phenol, $\mathrm{C}_{26} \mathrm{H}_{43} \mathrm{OH}, M=372$.

\begin{tabular}{|c|c|c|c|c|c|}
\hline$D$ & $P$ & $L$ & $M$ & $\begin{array}{l}\text { Procent- } \\
\text { gehalt der } \\
\text { Lösung }\end{array}$ & $\begin{array}{l}\text { Anziahl der } \\
\text { Moleküle Chole- } \\
\text { sterin in } \\
1000 \text { Molekiilen } \\
\text { Phenol }\end{array}$ \\
\hline $0 \cdot 17$ & 0.2558 & $33 \cdot 33$ & 343 & 0.76 & 2 \\
\hline $\int 0.37$ & 0.3178 & $16 \cdot 5$ & $395 \cdot 7$ & $1 \cdot 8$ & $4 \cdot 4$ \\
\hline$\{0 \cdot 52$ & $0 \cdot 4181$ & $16 \cdot 5$ & 370 & $2 \cdot 47$ & $5 \cdot 8\}$ \\
\hline 0.37 & $0 \cdot 3402$ & $16 \cdot 18$ & 432 & 2 & $5 \cdot 5)$ \\
\hline$\{0 \cdot 26$ & 0.228 & $16 \cdot 18$ & 412 & $1 \cdot 38$ & $3 \cdot 5$ \\
\hline 0.55 & 0.4644 & $16 \cdot 25$ & 394 & $2 \cdot 78$ & 7 \\
\hline 0.642 & 0.6525 & $17 \cdot 90$ & 431 & $3 \cdot 5$ & 9 \\
\hline $0 \cdot 734$ & $0 \cdot 6944$ & $16 \cdot 95$ & 433 & $3 \cdot 93$ & $12 \cdot 5$ \\
\hline
\end{tabular}

Die Bestimmung des Moleculargewichtes des Bilirubins durch Nencki and Rotschy kann, wie schon eingangs hervorgehoben, wegen der Zersetzlichkeit und Schwerlöslichkeit desselben nicht als entscheidend für die eine oder die andere Formel angesehen werden. Bekanntlich erhielt Maly durch Reduction des Bilirubins mittelst Natriumamalgam das Hydrobilirubin, identisch mit dem ron Jaffé aus Harn erhaltenen Urobilin. Die gut stimmenden Analysen Maly's entsprechen der Formel $\mathrm{C}_{32} \mathrm{H}_{40} \mathrm{~N}_{4} \mathrm{O}_{7}$. Und gerade die Zusammensetzung des Hydrobi- 
lirubins war für Maly eine Veranlassung, die Städeler'sche Formel des Bilirubins zu verdoppeln, da dann die Bildung des Reductionsproductes aus dem Bilirubin sich auf die einfachste Weise ergibt:

$$
\mathrm{C}_{32} \mathrm{H}_{36} \mathrm{~N}_{4} \mathrm{O}_{6}+\mathrm{H}_{2}+\mathrm{H}_{2} \mathrm{O}=\mathrm{C}_{32} \mathrm{H}_{40} \mathrm{~N}_{4} \mathrm{O}_{7} \text {. }
$$

Nun hat Hydrobilirubin die gute Eigenschaft, sich sowohl in Eisessig, wie in Phenol leicht zu lösen, und es war von hohem Interesse, zu sehen, ob dieser Farbstoff, dessen aus der Elementaranalyse abgeleitete Formel nicht theilbar ist, dem Raoult'schen Gesetze folgen wuirde. Auf die Bitte von Prof. Nencki hatte Herr Prof. Maly die grosse Freundlichkeit, das Hydrobilirubin rein darzustellen und mir etwas über $1.5 \mathrm{~g}$ zu übersenden. Das Präparat war zuletzt in Ammoniak gelöst und mit $\mathrm{HCl}$ gefällt. Ich habe es vor dem Gebrauch über $\mathrm{H}_{2} \mathrm{SO}_{4}$ im Vacuum bis zur Gewichtsconstanz getrocknet. Nach einigen Vorversuchen über die Löslichkeit des Farbstoffes habe ich folgende Bestimmungen in Phenol ausgeführt.

Hydrobilirubin $\mathrm{C}_{32} \mathrm{H}_{40} \mathrm{~N}_{4} \mathrm{O}_{7}, M=592$.

\begin{tabular}{|c|c|c|c|c|c|}
\hline$D$ & $P$ & $L$ & $\begin{array}{c}M, \\
\text { gefunden }\end{array}$ & $\begin{array}{c}\text { Procent- } \\
\text { gehalt der } \\
\text { Lösung }\end{array}$ & $\begin{array}{c}\text { Anzahl der } \\
\text { Molekiule Hydro- } \\
\text { bilirubin auf } \\
1000 \text { Molekiile } \\
\text { Phenol }\end{array}$ \\
\hline \hline 0.20 & $0 \cdot 212$ & $16 \cdot 77$ & 480 & $1 \cdot 2$ & 2 \\
0.42 & $0 \cdot 4117$ & $18 \cdot 14$ & 410 & $2 \cdot 2$ & $3 \cdot 5$ \\
0.65 & 0.6208 & $\mathbf{1 3 . 2 6}$ & 550 & $4 \cdot 4$ & $7 \cdot 4$ \\
\hline
\end{tabular}

Aus den mit Cholesterin und Cholalsäure erhaltenen Zahlen geht zunächst hervor, dass das Moleculargewicht dieser beiden Körper der einfachen Formel entspricht. Sodann zeigen meine Versuche, dass sehr verdünnte Lösungen in Phenol ganz abweichende Zahlen ergeben. So wurde für die Cholalsäure bei einer $0.7 \%$ igen Lösung und einer Depression von $0.166^{\circ}$ $M=395$ statt 502 gefunden. Erst eine einprocentige Lösung 
ergab einen dem richtigen Moleculargewichte nahestehenden Werth. Noch auffallender ist dies bei Hydrobilirubin, wo selbst eine $2 \cdot 2 \%$ ige Lösung und eine Depression von $0.42^{\circ}$ eine viel zu niedrige Zahl ergab. Erst als ich fast gesättigte Lösungen des Farbstoffes in Phenol anwendete, erhielt ich der Formel $\mathrm{C}_{32} \mathrm{H}_{40} \mathrm{~N}_{4} \mathrm{O}_{7}$ naheliegende Zahlen. Nencki und Rotschy haben allerdings ebenfalls fast gesättigte Lösungen des Bilirubins in Phenol angewendet und dabei für diesen Farbstoff Werthe erbalten, welche ziemlich der einfachen Formel entsprechen. Doch ist eben die Löslichkeit des Bilirubins in Phenol mehr wie zwölfmal geringer und es bleibt eine offene Frage, ob solche verdünnte Lösungen in Phenol dem Ra o ult'schen Gesetze folgen. Ein weiterer Unterschied zwischen der Cholalsäure und dem Cholesterin besteht darin, dass, während das letztere bei wachsender Concentration höbere Zahlen für das Moleculargewicht ergibt, die Cholalsäure selbst bei einer Concentration von 15 bis 20 Molekülen auf 1000 Molekiule Phenol und dementsprechend einer Depression von $0.8-2^{\circ}$ stets um ein Geringes kleinere Zahlen, als wie das theoretisch berechnete Moleculargewicht ergibt.

So wichtig und einfach die Raoult'sche Methode zur Bestimmung des Moleculargewichtes selbst complicirt zusammengesetzter, nicht fliuchtiger Substanzen ist, so kann sie doch nur verwendet werden am zu entscheiden, ob die aus der Elementaranalyse hervorgehende einfachste Formel oder ein Multiplum desselben dem wahren Moleculargewichte der betreffenden Verbindung entspricht. Bei Substanzen von hohem molecularem Gewichte, wo plus oder minus $\mathrm{H}_{2}, \mathrm{CH}_{2}$ oder $\mathrm{H}_{2} \mathrm{O}$ nur wenig die procentische Zusammensetzung beeinflusst, wird man durch die Raoult'sche Methode wegen der weiten Fehlergrenzen keine definitive Aufklärung erhalten, wie dies ubrigens auch schon von anderen Experimentatoren hervorgehoben worden ist. So hat z. B. Latschin off, wie schon erwähnt, die Formel $\mathrm{C}_{25} \mathrm{H}_{42} \mathrm{O}_{5}$ für die Cholalsäure statt der von Strecker ermittelten und auch von anderen Chemikern, die die Gallensäuren untersuchten, adoptirten Formel $\mathrm{C}_{24} \mathrm{H}_{40} \mathrm{O}_{5}$, aufgestellt. Die von mir erhaltenen Zahlen, sowohl in Eisessig wie in Phenol, stehen näher der Streckerschen Formel. Es wäre jedoch unzulässig, einzig auf Grund 
dieser Bestimmungen die Lats chin off'sche Formel zu verwerfen. Die Raoult'sche Methode wird in der Zukunft immer mehr angewendet. Es ist jedoch nöthig, sich dabei nicht auf ein einziges Lösungsmittel zu beschränken, sondern womöglich mehrere zu verwenden. Man muss sich auch stets vergegenwärtigen, dass der Raoult'sche Werth $\boldsymbol{T}$ bei einem und demselben Lösung:smittel für verschiedene Körperklassen nicht immer denselben Werth hat und dass die Concentration der Lösungen stets berücksichtigt werden muss.

Der Werth $\boldsymbol{T}$, d. h. die "moleculare Depression", kann, wie van t'H off gezeigt hat, aus der latenten Schmelzwärme und der absoluten Schmelztemperatur abgeleitet werden. Will man für eine Klasse von Verbindungen den Werth $T$ empirisch bestimmen, so verfährt man am besten nach den Angaben von Raoult, indem man sich eine Curve anlegt, auf deren Abscissen die Depressionen, auf deren Ordinaten die Werthe für den Erniedrigungscoëfficienten - den Werth $A$ in der Raoult'schen Formel - aufgetragen werden. 